Publications of the Astronomical Society of the Pacific, 117:996-1003, 2005 September

(C) 2005. The Astronomical Society of the Pacific. All rights reserved. Printed in U.S.A.

\title{
The Very Large Telescope Visible Multi-Object Spectrograph Mask Preparation Software
}

\author{
D. Bottini ${ }^{1}$ B. Garilli, ${ }^{1}$ D. Maccagni, ${ }^{1}$ L. Tresse, ${ }^{2}$ V. Le Brun, ${ }^{2}$ O. Le Fèvre, ${ }^{2}$ J. P. Picat, ${ }^{3}$ R. Scaramella, ${ }^{4}$ \\ M. Scodeggio, ${ }^{1}$ G. Vettolani, ${ }^{4}$ A. Zanichelli, ${ }^{4}$ C. Adami, ${ }^{2}$ M. Arnaboldi, ${ }^{5}$ S. Arnouts, ${ }^{2}$ S. Bardelli, ${ }^{6}$ M. Bolzonella, ${ }^{7}$ \\ A. Cappi, ${ }^{6}$ S. Charlot,${ }^{8,9}$ P. Ciliegi,${ }^{6}$ T. Contini, ${ }^{3}$ S. Foucaud,${ }^{1}$ P. Franzetti, ${ }^{1}$ L. Guzzo, ${ }^{10}$ O. Ilbert ${ }^{6}$ A. Iovino, ${ }^{10}$ \\ H. J. McCracken, ${ }^{9}, 11$ B. Marano, ${ }^{7}$ C. Marinoni, ${ }^{10}$ G. Mathez, ${ }^{3}$ A. Mazure, ${ }^{2}$ B. Meneux, ${ }^{2}$ R. Merighi, ${ }^{6}$ S. Paltani, ${ }^{2}$ \\ A. Pollo, ${ }^{10}$ L. Pozzetti, ${ }^{6}$ M. Radovich, ${ }^{5}$ G. Zamorani, ${ }^{6}$ and E. ZucCa ${ }^{6}$ \\ Received 2004 December 23; accepted 2005 May 24; published 2005 July 28
}

\begin{abstract}
VIMOS (Visible Multi-Object Spectrograph) is a multiobject imaging spectrograph installed at the VLT (Very large Telescope) at the ESO (European Southern Observatory) Paranal Observatory that is especially suited for survey work. VIMOS is characterized by its very high multiplexing factor: it is possible to take up to 800 spectra with 10" long slits in a single exposure. To fully exploit its multiplexing potential, we designed and implemented a dedicated software tool: the VIMOS Mask Preparation Software (VMMPS), which allows the astronomer to select the objects to be spectroscopically observed, and provides for automatic slit positioning and slit number maximization within the instrumental constraints. The output of VMMPS is used to manufacture the slit masks to be mounted in the instrument for spectroscopic observations.
\end{abstract}

\section{INTRODUCTION}

VIMOS (Visible Multi-object Spectrograph) is a survey multiobject spectrograph that has been operational at one of the Nasmyth foci of the Melipal telescope of the ESO (European Southern Observatory) VLT (Very Large Telescope) since 2003 April. VIMOS was designed and built by the French-Italian VIRMOS Consortium (Le Fèvre et al. 2000). VIMOS is based on a classical focal reducer design replicated in four separate channels operating simultaneously (see Fig. 1). It has three operational modes: imaging, with broadband UBVRIz filters; MOS (multi-object spectroscopy), with a choice of five grisms giving spectral resolutions from 200 to 2500 in the wavelength range $0.37-1 \mu \mathrm{m}$; and IFS (integral

\footnotetext{
${ }^{1}$ IASF-INAF, via Bassini 15, I-20133, Milano, Italy.

${ }^{2}$ Laboratoire d'Astropysique de Marseille, UMR 6110 CNRS, Université de Provence, BP8, 13376 Marseille Cedex 12, France.

3 Laboratoire d'Astrophysique de l'Observatoire Midi-Pyrénées (UMR 5572), 14 Avenue Edouard Belin, F-31400 Toulouse, France.

${ }^{4}$ IRA-INAF, Via Gobetti, 101, I-40129, Bologna, Italy.

5 INAF-Osservatorio Astronomico di Capodimonte, Via Moiariello 16, I-80131, Napoli, Italy.

${ }^{6}$ INAF-Osservatorio Astronomico di Bologna, Via Ranzani,1, I-40127, Bologna, Italy.

${ }^{7}$ Università di Bologna, Dipartimento di Astronomia, Via Ranzani,1, I-40127, Bologna, Italy.

${ }^{8}$ Max Planck Institut fur Astrophysik, 85741, Garching, Germany.

${ }^{9}$ Institut d'Astrophysique de Paris, UMR 7095, 98 bis Boulevard Arago, 75014, Paris, France.

${ }^{10}$ INAF-Osservatorio Astronomico di Brera, Via Brera 28, Milan, Italy.

${ }^{11}$ Observatoire de Paris, LERMA, 61 Avenue de l'Observatoire, 75014, Paris, France.
}

field spectroscopy), whereby 6400 fibers coupled with microlenses produce spectra of a contiguous area of $54^{\prime \prime} \times 54^{\prime \prime}$.

In MOS mode, observers design four slit masks (one for each quadrant) from images taken prior to the spectroscopy observing run (hereafter called "preimages") and a user-supplied source catalog. Then the masks are manufactured by the MMU (mask manufacturing unit), which is a dedicated lasercutting machine (Conti et al. 1999, 2001). Once inserted in the focal plane, masks are aligned on the sky before the exposure.

One of the main drivers in the design of VIMOS was the multiplexing factor; i.e., the possibility of packing as many spectra as possible on the detectors. For example, at the limiting magnitude $I<24$, with a low-resolution grism, we have been able to obtain up to 1000 spectra.

The number of spectra that can be packed on a detector depends on the detector size, the length of the spectra, the length of the slits, the possibility of placing slits over the whole field of view without losing wavelength coverage, and last but not least, the spatial distribution of the targets. To maximize the number of slits, we have adopted a similar approach to that successfully used by the Canada-France Redshift Survey (CFRS) on the MOS/SIS (Multi-Object Spectrograph and Subarcsecond Imaging Spectrograph) at the Canada-FranceHawaii 3.6 m Telescope (CFHT; Le Fèvre et al. 1994), allowing zero-order and second-order spectra to overlap with the firstorder spectra from other slits. This makes for an efficient packing of spectra on the detectors, with several superimposed banks of spectra, and with strict constraints on slit mask design and data processing (Le Fèvre et al. 1995).

To improve on this concept, the VIMOS optical design is such that slits can be placed anywhere in the field of view, and 


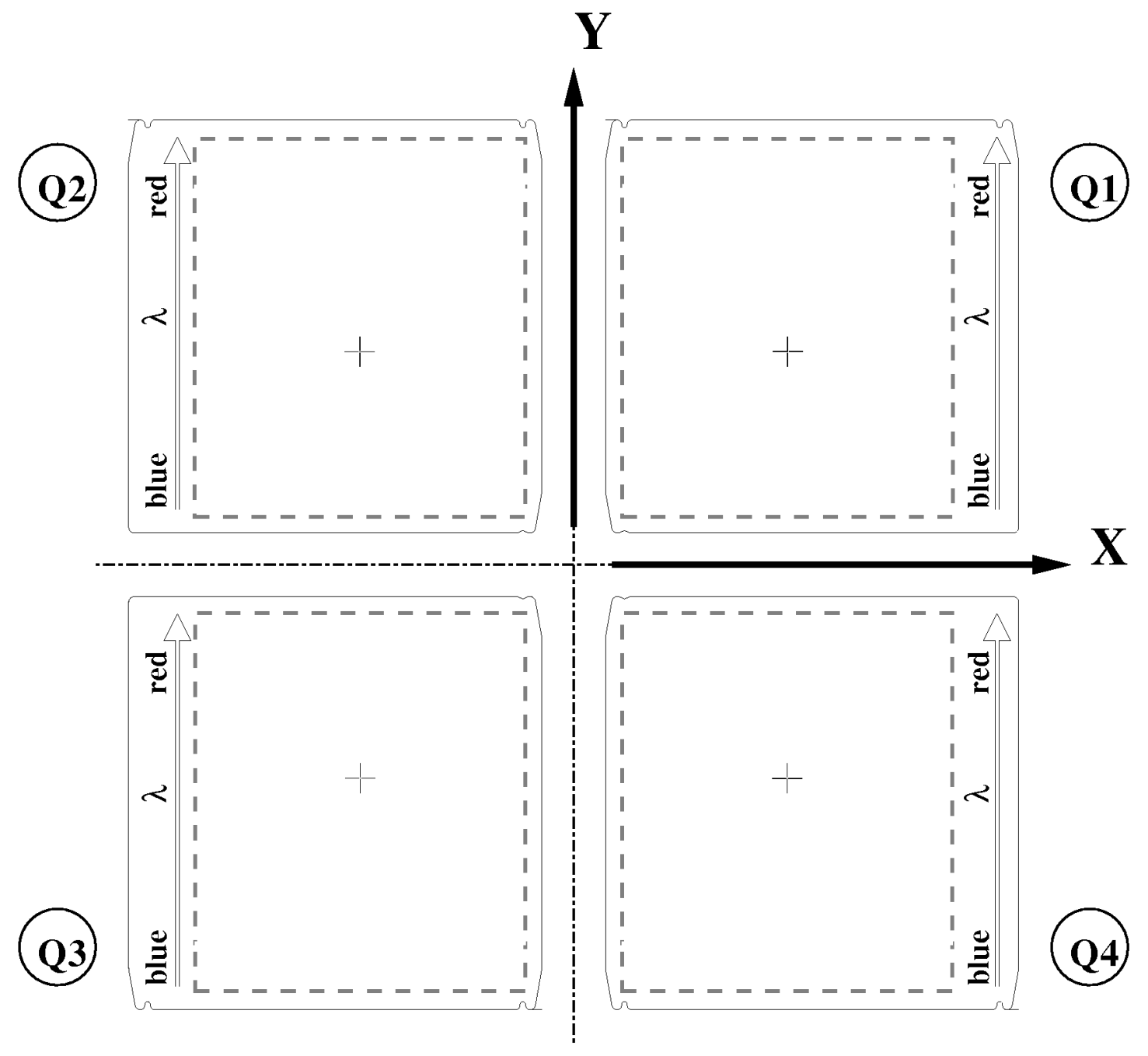

FIG. 1.-Location of the four masks in the VIMOS quadrants in the correct relative scale. The outer lines define the contours of the four masks. The dispersion direction of the spectra is along the $Y$ axis. The four crosses mark the position of the optical axis of each quadrant, while the dashed line indicates the $244 \times 279$ mm field of view projected onto the four CCD detectors (for more details, see Conti et al. 2001).

the optical cameras image the spectra on $2048 \times 4096$ pixels $^{2}$, the 4096 pixels being placed along the dispersion direction, while the field of view on the sky is imaged only on $2048 \times 2340$ pixels $^{2}$. This means that for any slit placed in the field of view, the full wavelength range of a low-resolution spectrum is recorded on the detector. This property also applies to higher resolution $R \sim 2500$ spectra, which are fully recorded on the detector for most slit positions in the field of view.

The large number of slits (up to 200 per quadrant) involved in the VIMOS spectroscopic observations, together with slitpositioning constraints, makes it practically impossible for the astronomer to manually choose and place all the slits, in particular if one wants to maximize the number of slits. The software tool we are describing in this paper, called the VIMOS Mask Preparation Software (VMMPS), allows for the design of the slit masks, taking into account all the instrument constraints and solving the problem of maximizing the multiplexing factor for a certain target field (Garilli et al. 1999). This tool has been delivered by our Consortium to ESO and is now distributed by ESO to all successful proposers.

VMMPS provides the astronomer with tools for the selection of objects to be spectroscopically observed. It includes interactive object selection, it handles curved slits, and it uses an algorithm for automatic slit positioning that derives the most effective solution in terms of number of objects selected per field. The slit-positioning algorithm takes into account both the initial list of user's targets and the constraints imposed either by the instrument characteristics and/or by the requirement of easily reducible data. The number of possible slit combinations in a field is in any case very high, and the task of slit maxi- 
mization cannot be solved through a purely combinational approach. We have introduced an innovative approach based on the analysis of the function $N_{\text {slit }} / W_{\text {col }}$, where $N_{\text {slit }}$ is the number of slits within a column and $W_{\text {col }}$ is the width of the column. The algorithm has been fully tested and validated.

In this paper we describe the MOS observation preparation process and imaging and catalog handling ( $\$ 2$ ), the instrumental and data-reduction requirements $(\$ 3)$, and the slit-positioning optimization code $(\S 4)$. We conclude in $\S 5$.

\section{PREPARATION OF VIMOS MOS OBSERVATIONS}

We must distinguish two cases: (1) the spectroscopic targets are derived from an image taken with VIMOS itself in imaging mode, and thus the targets have VIMOS internal coordinates; (2) the spectroscopic targets are to be chosen from a catalog (which we call the user catalog) with good astrometry but obtained with another instrument/telescope.

In principle, telescope (VLT) and site (Paranal) characteristics make it conceivable to use slits as narrow as 0.5 , a possibility that in turn requires an excellent mapping of the projection of the sky onto the focal plane. The mapping of the focal plane onto the CCDs is part of the calibration procedure of the instrument, and it has been proved that the accuracy of this calibration is better than 1 pixel $(0.2)$ at any point in the field of view. The mapping of the sky onto the CCD, or the astrometric calibration of the images, is a more difficult task, where an accuracy of 0.3 (absolute astrometry) is commonly considered as an excellent result. Relative astrometry (i.e., the relative position of objects within the field of view) is usually much more accurate ( 0 ". 1 or less).

For the case in which the target object coordinates are already in VIMOS pixels (case $a$ ), the computation of slit coordinates requires only the well-known CCD-to-focal-plane calibration. Otherwise (case $b$ ), a tool is needed to transform the user catalog celestial coordinates into VIMOS coordinates. This is accomplished by first obtaining a "preparatory image" with VIMOS, which will have a moderately accurate astrometric solution. From this image, a catalog of objects with $X$ and $Y$ coordinates in VIMOS pixels and approximate sky coordinates can be obtained with any detection algorithm. The first step that VMMPS performs is the cross-correlation and the subsequent transformation to the coordinate grid defined by the VIMOS image. The cross-correlation algorithm finds common objects within a given tolerance between the user catalog and the VIMOS preimage catalog. The output is a catalog of objects with the original celestial coordinates and the $X$ and $Y$ VIMOS coordinates. The matching and coordinate transformation processes can be iterated until the requested $\mathrm{rms}$ is reached. The algorithm is based on the WCS (world coordinate system) libraries, and the coordinate transformation is done by fitting the parameters of the $\mathrm{CO}$ matrix with a polynomial, using the WCS fitplate function.

In this way, errors in the VIMOS coordinates are confined within the errors of the user catalog internal relative astrometry and of the final transformation. The preparatory image is not required to be so deep that it detects all the spectroscopic targets. It should, however, be deep enough to provide enough targets for a cross-correlation between the catalog extracted from the image itself and the user catalog. The coordinate transformation can be very accurate, provided that it is computed using a sufficient number of objects. Tests on real data have shown that $60-80$ objects in common between the preimaging and the user catalogs give excellent results in terms of positional accuracy of the solution. Any possible rigid offset between the solution found and the absolute pointing will be corrected for at the telescope, with the fine-pointing procedure given below; this can correct for offsets as large as $3^{\prime \prime}$.

The next step is to define the catalog "special" objects. These are the objects to be used for mask alignment (reference), the objects that must be observed in any case (compulsory), and the objects you do not want to observe (forbidden). Eventually, "special" tilted or curved slits can be defined interactively. The last step is to define the set of objects to be observed. This step is performed by SPOC (Slit Positioning Optimization Code), which is the core of VMMPS. SPOC places slits, maximizing their number in a VIMOS quadrant and taking into account special objects, special slits, and slit-positioning constraints.

\section{INSTRUMENTAL AND DATA-REDUCTION REQUIREMENTS}

\subsection{Masks}

The VIMOS focal plane is divided into four quadrants, thus four masks (one mask set) are needed for each MOS observation. The scale at the focal plane where masks must be inserted is $0.578 \mathrm{~mm}$ arcsec ${ }^{-1}$, and each quadrant has a field of view of about $7^{\prime} \times 8^{\prime}$. This defines the gross dimensions of the masks and, together with the expected best seeing at the VLT ( 0".3), the minimum slit width.

Figure 1 shows an outline of the VIMOS focal plane with its reference system. The size of a mask is $305 \times 305 \mathrm{~mm}$, and the contour geometry is determined by the mechanical interface with the focal plane and with the automatic maskinsertion mechanism (Conti et al. 2001).

It is important to note that the relative position of the masks is fixed and cannot be changed. Therefore, when preparing masks, the relative positions of the targets must be consistent over the whole field of view, and not only over a single quadrant.

\subsection{Slit-Positioning Constraints}

Within a limiting magnitude of $I=24$, more than 1000 objects can be present in a VIMOS quadrant. Not all of these objects can be spectroscopically observed in a single exposure, because the overlap of spectra, both in the spatial and in the dispersion direction, must be avoided. When low-dispersion 


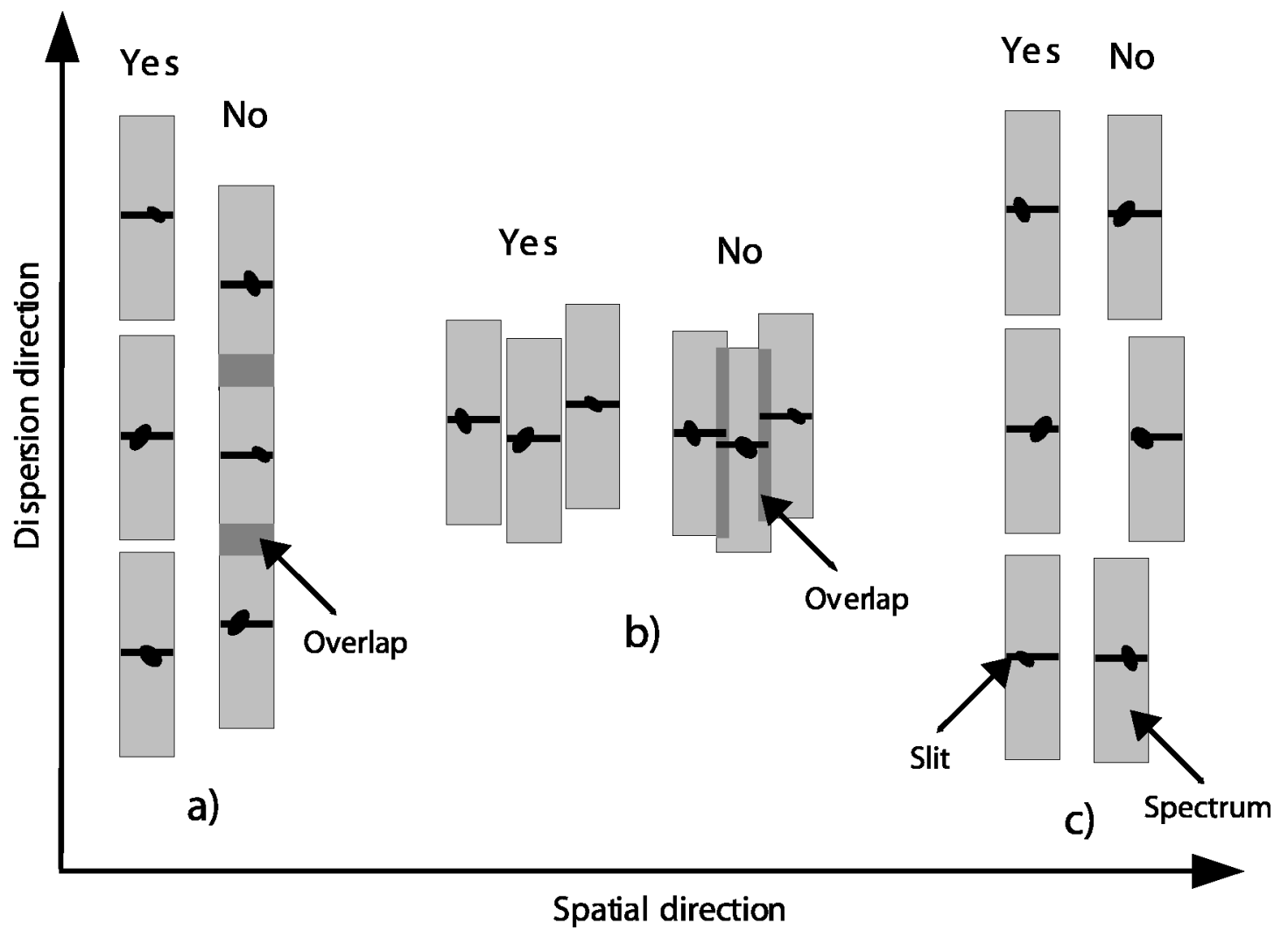

FIG. 2.-Spatial constraints for MOS slit positioning: "Yes" indicates allowed slit placements, "No" indicates forbidden slit placements. Case $a$ : slits must not overlap in the dispersion direction; case $b$ : slits must not overlap in the spatial direction; case $c$ : slits must be exactly aligned along the dispersion direction (see $\S 3.1$ ).

grisms are used, there can be several spectra along the dispersion for the same position on the CCD $X$ coordinate (multiplexing). In this case, objects must have a separation greater than their first-order spectrum length (see Fig. 2, case $a$ ), and the spectra resulting from slits placed on objects at different spatial coordinates must not overlap (see Fig. 2, case $b$ ). Furthermore, low-resolution grisms produce second-order spectra that, although of very low intensity ( 3\% of the intensity of the first order), can be extremely annoying for the sky subtraction of the faintest objects. For a better estimate of the spectral background, it is preferable to have second-order spectra overlapping any eventual first-order spectrum in an exact way; i.e., the two slits placed "one above the other" should be of the same length and aligned (see Fig. 2, case c). VIMOS optical distortions are very small, and aligned slits on the masks always produce well-aligned spectra on the CCD.

This requirement ensures that the second-order sky contamination of the slit below, and the zero order of the slit above (in the dispersion direction), can be removed together with the first-order sky when the dispersed background of a slit is processed. This process does not remove the second and zero orders of the objects in the slits below/above, but it completely eliminates the inconvenience of having to remove a step-func- tion contamination along the spatial direction of the slits when correcting for the sky background.

On the contrary, VIMOS high-dispersion grisms produce first-order spectra approximately as long as the dispersion direction of a VIMOS quadrant; i.e., only a single column of slits can be placed along the dispersion direction.

The slit length is set by the object size plus a minimum area of sky on both sides of the object, required for a stable fitting of the sky signal to be removed during data processing. The size of this area should be of the order of $2^{\prime \prime}$ per side (i.e., about 10 pixels) to ensure a good sky-level fitting.

Slits as narrow as 0"5 are a real possibility for VIMOS, which implies an extremely precise pointing and centering of objects in slits. The best and safest way to obtain such accuracy is to have reference objects in the field to refine the pointing on the sky. Once the telescope is on the field, an exposure is taken with the mask inserted, and pointing is refined so that reference objects fall exactly at the center of the reference "holes." Reference objects must be bright and pointlike, and they must be part of the same user-input catalog. Reference objects have to be manually chosen. The slit-positioning algorithm has to handle them in a special way, as they have square holes with a fixed size instead of rectangular slits. Although in principle 


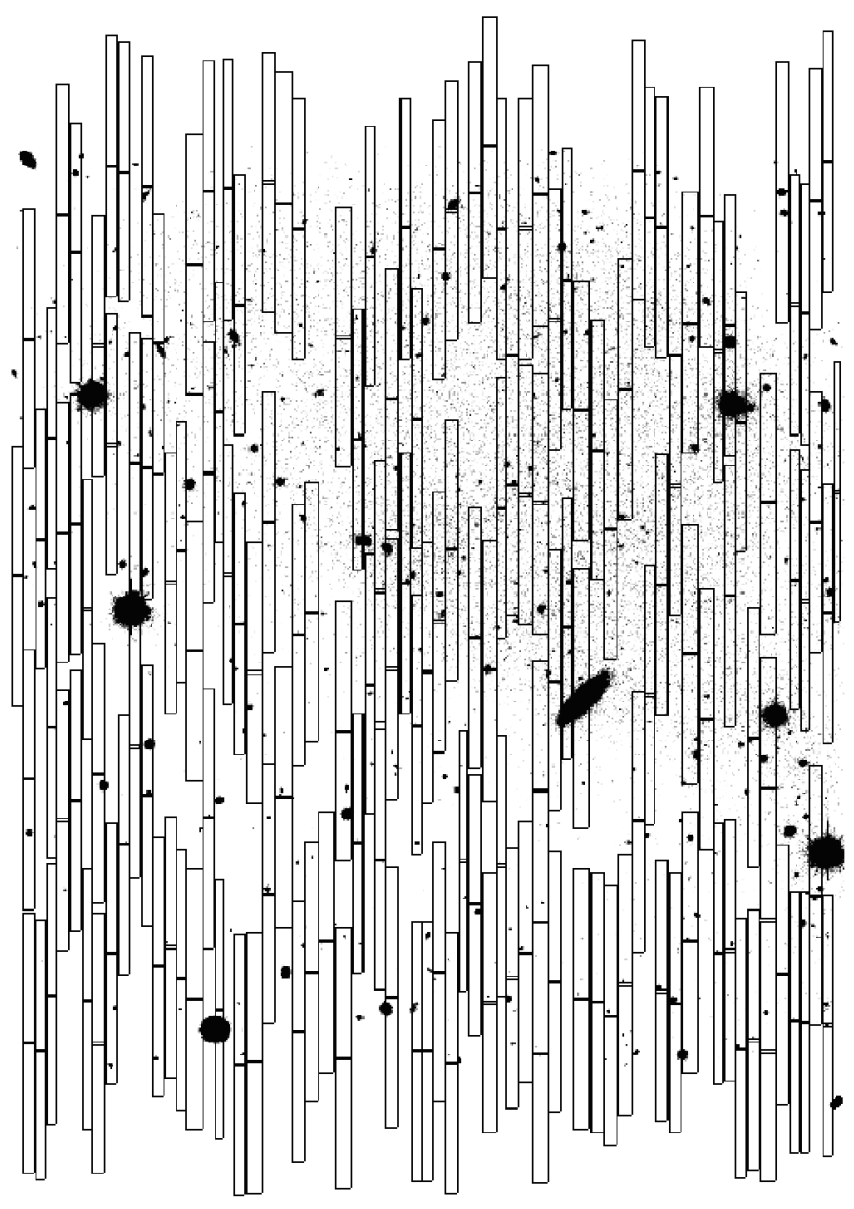

FIG. 3.-Output of the VMMPS/SPOC algorithm run on a single quadrant of a VIMOS image and using an external user catalog; the number of slits positioned is maximized by VMMPS, satisfying all the user and instrument constraints. Slit and spectrum contours are plotted over the astronomical image.

only one reference object per quadrant would be required for pointing refining, a minimum of two reference objects per quadrant is recommended.

As reference objects are bright, their second-order spectrum contamination is strong. For this reason, no slit is allowed in the same spatial coordinate range as the reference object holes. The same requirement is applied to curved or tilted slits for particularly extended and interesting objects, and no spectrum overlapping is thus allowed.

These constraints must be fully taken into account by any slit-positioning code.

\subsection{Manual Selection of Objects}

VMMPS is based on the astronomical package SKYCAT, distributed by ESO. SKYCAT has enhanced real-time display capabilities and provides basic functions for catalog handling. Display and catalog functions are coupled with good overlay capabilities, and WCS is well supported. The VMMPS graphical user's interface is a SKYCAT plug-in developed in Tcl/Tk. Allowed formats are FITS for images and ASCII for catalogs.

As slits are positioned with an automated procedure that maximizes the number of placed slits, there is no way to choose objects a priori. For all scientific purposes that do not rely on systematic surveys, this can be a disadvantage. Compulsory objects are the answer to such needs: the automatic procedure places slits on them (after having checked that they do not violate the constraints described above) before anything else, then it tries to place additional slits on objects randomly chosen from the catalog.

Forbidden objects are the opposite of compulsory objects; they are flagged by the user so that no slit is placed on them.

Last, but not least, a number of scientific programs would greatly benefit from having curved or tilted slits that better follow the object profile (gravitational arcs are just the most obvious example). The laser-cutting machine (Conti et al. 2001) is capable of cutting arbitrary shapes. Therefore, VMMPS allows the user to interactively design curved or tilted slits. Curved slits are defined by fitting a Bezier curve to a set of points interactively chosen by the user.

\section{SLIT POSITIONING OPTIMIZATION CODE (SPOC)}

The large number of objects and slits involved in the VIMOS spectroscopic observations, together with the positioning constraints and the particular objects described above, make it practically impossible for the astronomer to manually choose and place the slits. For this reason, a tool for the automatic positioning of slits and the maximization of their number has been implemented: the Slit Positioning Optimization Code (SPOC), which is the core of VMMPS. Given a catalog of objects, it maximizes the number of observable objects in a single exposure and computes the corresponding slit positions. SPOC places slits on the field of view, taking into account special objects (reference, compulsory, and forbidden), special slits (curved, tilted, or in user-defined dimensions), and slitpositioning constraints. An example of SPOC slit positioning for a VIMOS quadrant is shown in Figure 3.

The issue to be solved is a combinatory computational problem. Due to the constraint of slits aligned along the dispersion direction, the problem can be slightly simplified: the quadrant area is considered as a sum of columns that are not necessarily of the same width in the spatial direction. Slits within the same column have the same length, so that the alignment of orders is fully ensured. The problem is thus reduced to being a monodimensional one. It is easy to show that the number of combinations is roughly given by $N_{c}=N_{w}^{N_{\text {col }}}$, where $N_{c}$ is the number of combinations, $N_{w}$ the number of possible column widths, and $N_{\text {col }}$ the average number of columns.

The slit length (or column width) can vary from a minimum of 4" (20 pixels; i.e., twice the minimum sky region required for the sky subtraction) to a maximum of $30^{\prime \prime}$ (150 pixels; limit 
imposed by the slit laser-cutting machine) in 1 pixel steps. The average number of columns is estimated as the spatial direction size of the field of view, divided by the most probable slit length, typically 50 pixels $\left(10^{\prime \prime}\right)$; i.e., $2048 / 50=41$. The number of combinations is then $N_{c}=(130)^{41} \sim 4.7 \times 10^{86}$, corresponding to about $10^{60}$ years of CPU work.

The problem is similar to the well-known traveling salesman problem. In the standard approach, it is solved by randomly extracting a "reasonable" number of combinations and maximizing over this subsample. In our case, due to computational time, the "reasonable" number of combinations to be processed cannot be higher than $10^{8}-10^{9}$. As this number is much smaller than the total number of possible combinations, the resulting maximization cannot be a good approximation of the real maximum.

Our approach has been to consider only the most probable combinations; i.e., the ones that have the highest probability to maximize the solution. For each spatial coordinate, we vary the column width from the given minimum to the given maximum, and we count how many objects can be placed in the column. Figure 4 shows the number of slits in a column, divided by the column width $\left(N_{\text {slit }} / W_{\text {col }}\right)$, as a function of the column width. It is obvious that increasing the column width does not monotonically increase the number of slits that can be placed in a column. However, the function has evident maxima, which of course depend on the size of the objects. For each spatial coordinate, only the column widths corresponding to the five peaks are worth considering, as they correspond to local maxima of the number of slits per column. The exact positioning of the peaks varies for each spatial coordinate, but the shape of the function remains the same. Using a partition exchange method, the position of the peaks can be easily found in no more than six or seven trials.

For each spatial coordinate, we have $K$ (where $K$ is the number of peaks; in our example, $K=4$ ) possible columns with their own length and number of slits. Although the number of combinations to be tested has been decreased (in our example, about $4^{41}=4.8 \times 10^{24}$ ), it is still too large in terms of computational time.

A further reduction can be obtained if, instead of simultaneously considering all the columns, we consider sequentially $M$ subsets of $N$ consecutive columns, which cover the whole quadrant. At this point, we should vary $N$ (and consequently $M$ ) to find the best solution. In practice, when $N$ is higher than 8 10 , nothing changes in terms of number of observable objects. For $N=10$ and thus $M=4$ [i.e., $2048 /(4 \times 50)$ ], the number of combinations is reduced to only $4 \times 4^{10}=4 \times 10^{6}$; that means a few seconds of CPU work.

As a consequence of the optimization process, small-sized objects are favored over the larger ones. Small objects are statistically fainter than big ones, so faint objects are slightly favored over the bright ones, and this can be a drawback for some observational projects.

To overcome this drawback, a less optimized algorithm has

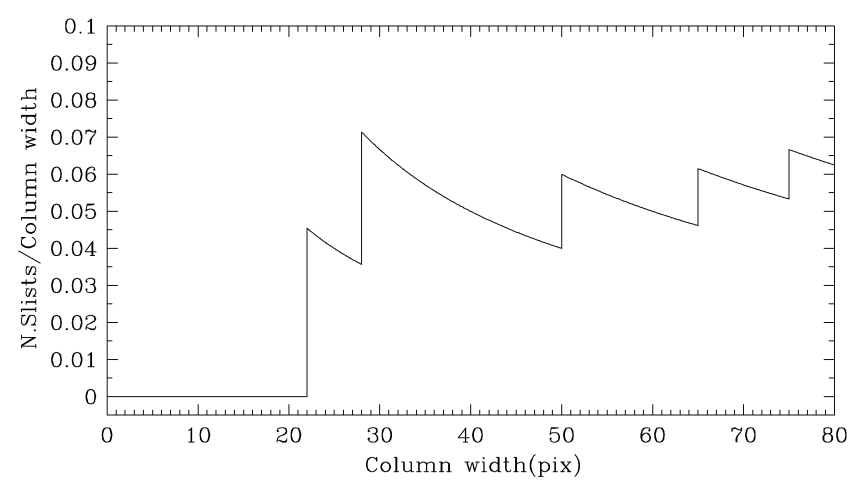

FIG. 4.-Number of slits in a column, divided by the column width $\left(N_{\text {slit }} / W_{\text {col }}\right)$, as a function of the column width. When the column width increases, this function decreases, but a maximum is reached every time a new slit is placed in the column itself. For small values of $W_{\text {col }}$, no slits can be placed, and therefore the function value is zero.

also been implemented in SPOC. This alternative algorithm does not optimize all columns simultaneously; rather, it builds the $N_{\text {slit }} /$ column $_{\text {width }}$ function (Fig. 4) column by column, without considering object sizes, and it takes only the maximum. Then it increases each column width to account for object sizes.

We tested these two different SPOC maximization modalities on a subsample of about 90,000 objects extracted from the VIMOS-VLT Deep Survey database (VVDS; Le Fèvre et al. 2004). The magnitude and the radius distributions of this subsample and of the SPOC output (about 7000 objects) are shown in Figure 5. The excess of small (Fig. 5, case $a$ ) and faint (Fig. 5, case $c$ ) objects produced by SPOC with the best optimization modality disappears when the less optimized modality is used (Fig. 5, cases $b$ and $d$ ), while the number of placed slits decreases by a few percent.

\section{CONCLUSIONS}

1. VMMPS provides the VIMOS observer with user-friendly software tools to select the spectroscopic targets in the instrument field of view, including interactive object selection, handling of curved slits, and maximization of the number of targets once the input catalog is defined. The VMMPS output is a set of files that are passed to the VIRMOS MMU to cut the slits in the masks and that are included in the file header of the spectroscopic images for the subsequent data reduction.

2. VMMPS has been successfully used by the VIMOS team for the preparation of the VIMOS-VLT Deep Survey (Le Fèvre et al. 2005), and by the European astronomical community for the preparation of their specific observation programs.

3. VMMPS is a general-purpose, rather than instrumentspecific, tool for multiobject spectroscopy, and its code has been distributed to GMOS (Gemini) and OSIRIS (Gran Telescopio Canarias). 


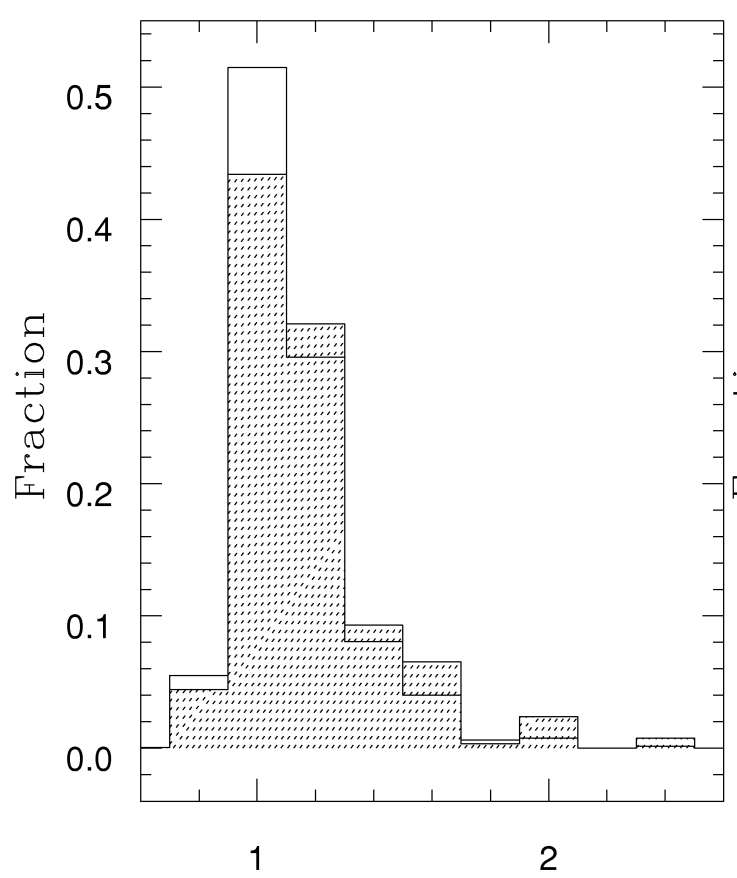

a)

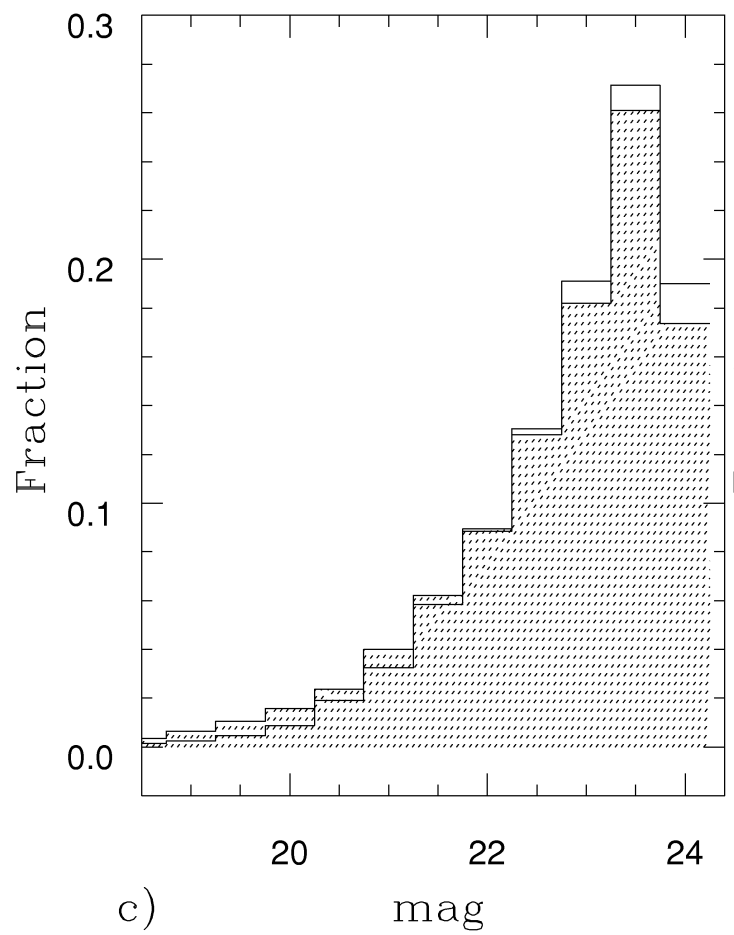

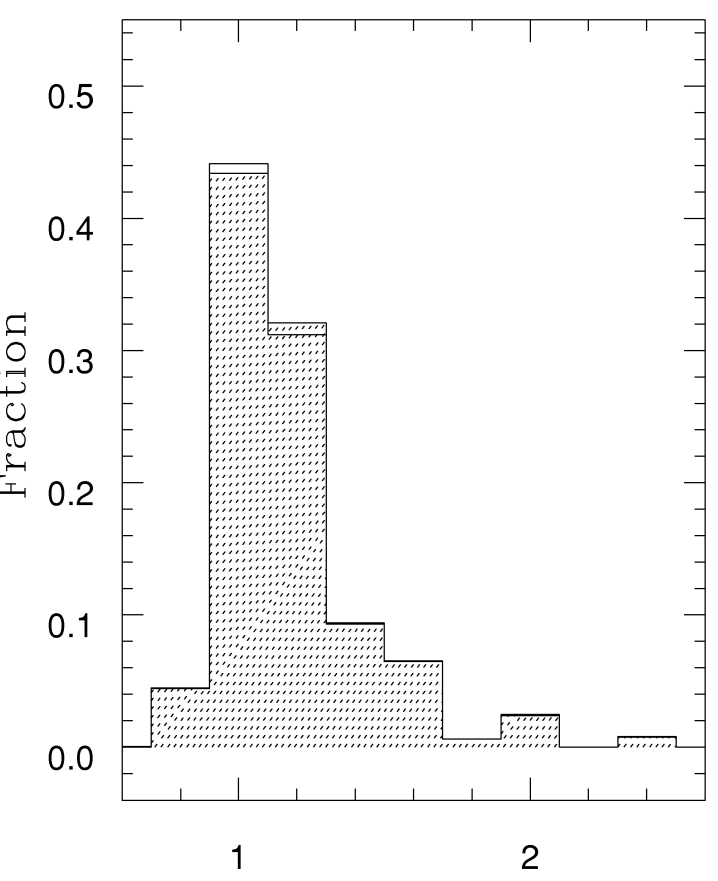

b)

$\operatorname{rad}(\operatorname{arcsec})$

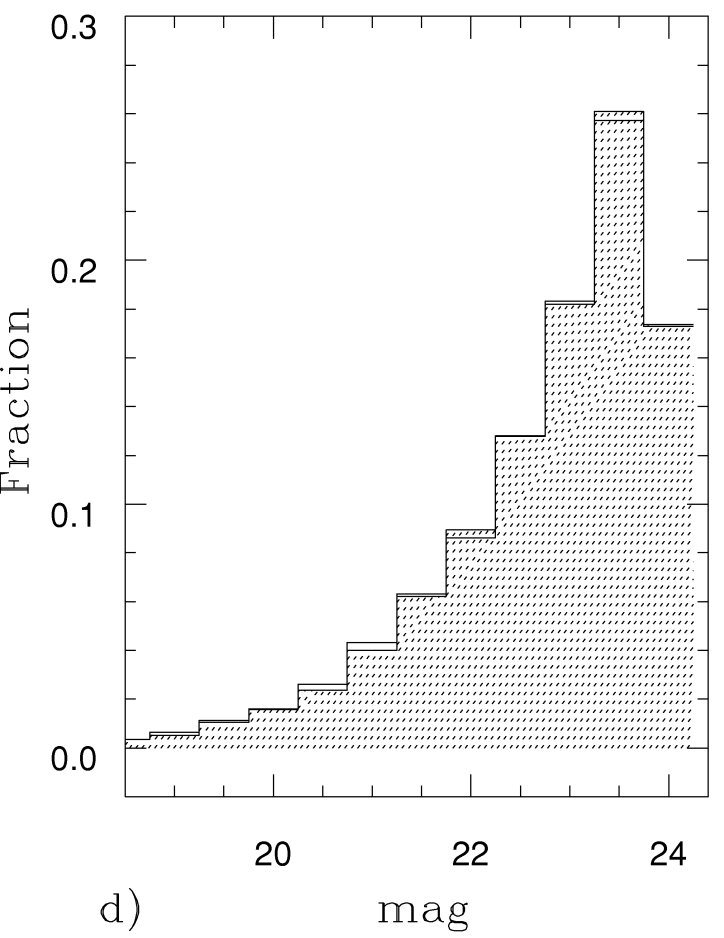

FIG. 5.-Comparison of two different SPOC-maximization modalities applied to a sample of about 90,000 objects. The figure shows the magnitude and the radius distributions of this sample (shaded histogram) and of the SPOC output. The excess of small (case $a$ ) and faint (case $c$ ) objects produced by SPOC with the best optimization modality disappears when the less optimized modality is used (cases $b$ and $d$ ), while the number of placed slits decreases by a few percent. 
The VMMPS has been developed under ESO contract 50979/ INS/97/7569/GWI. This research has been developed within the framework of the VVDS consortium. This work has been partially supported by the Italian Ministry (MIUR) grants COFIN2000 (MM02037133) and COFIN2003 (2003020150).

\section{REFERENCES}

Conti, G., Chiappetti, L., Mattaini, E., Maccagani, D., Le Fèvre, O., Saisse, M., \& Vettolani, G. 1999, in Conference on Telescopes, Instruments, and Data Processing for Astronomy in the Year 2000, S. Agata, Astro Tech. J., 2, 2

Conti, G., et al. 2001, PASP, 113, 452

Garilli, B., Bottini, D., Tresse, L., Le Fèvre, O., Saisse, M., \& Vettolani, G. 1999, in Conference on Telescopes, Instruments, and Data Processing for Astronomy in the Year 2000, S. Agata, Astro Tech. J., 2,2
Le Fèvre, O., Crampton, D., Felenbok, P., \& Monnet, G.,1994, A\&A, 282,325

Le Fèvre, O., Crampton, D., Lilly, S.J., \& Hammer, F., Tresse, L., 1995, ApJ, 455, 60

Le Fèvre, O., et al. 2000, Proc. SPIE, 4008, 546

- 2004, A\&A, 417, 839

2005, A\&A, in press 